\title{
Feasibility and Safety of Single-Incision Laparoscopic Appendectomy by a Surgical Resident under Supervision of a Staff Surgeon
}

\author{
Jung II Joo, M.D., Jung Ho Park, M.D., Dong Hyun Kim, M.D., Sang Woo Lim, M.D., Ph.D. \\ Department of Surgery, Hallym University College of Medicine, Hallym University Sacred Heart Hospital, Anyang, Korea
}

\begin{abstract}
Purpose: This study was aimed at reporting our experience with single-incision laparoscopic appendectomies (SILA) performed by a surgical resident, and to evaluate the safety and feasibility of the procedure, together with a comparison of the outcomes of the same procedure performed by a staff surgeon.
\end{abstract}

Methods: We conducted a retrospective case series analysis of 60 consecutive patients who underwent SILA. Two surgeons, an attending staff surgeon and a second-year surgical resident, performed the SILA procedures. SILA procedures performed by the resident were intraoperatively guided and supervised by the staff surgeon.

Results: A total of 60 case-matched patients with acute appendicitis underwent a SILA performed by either the resident or attending staff. There was no difference in patient demographics between the two groups of patients. The mean operation time was longer in the resident group than in the staff group (43.2 \pm 6.0 minutes vs. $32.9 \pm 10.5$ minutes, $p<0.001$ ). There was no significant difference in the operative data between the two groups. No conversion to an open procedure occurred in either group. Postoperative pain, time to onset of oral intake, and number of days of postoperative hospital stay were similar in both groups.

Conclusion: SILA procedures performed by a resident are safe and feasible despite longer operation times. Perioperative supervision and guidance by an attending staff surgeon may facilitate surgical outcomes.

Keywords: Laparoscopy, Appendectomy, Resident, Education, Training
Received August 17, 2018

Revised 1st September 4, 2018

2nd September 19, 2018

3rd September 21, 2018

Accepted September 28, 2018

Corresponding author

Sang Woo Lim

Department of Surgery, Hallym University College of Medicine, Hallym University Sacred Heart Hospital, 22 Gwanpyeong-ro 170-gil, Dongan-gu, Anyang 14068, Korea Tel: $+82-31-380-3772$

Fax: +82-31-380-1619

E-mail: swlim12@hallym.ac.kr ORCID:

https://orcid.org/0000-0001-8248-8406

This is an Open Access article distributed under the terms of the Creative Commons Attribution Non-Commercial License (http:// creativecommons.org/licenses/by-nc/4.0/) which permits unrestricted non-commercial use, distribution, and reproduction in any medium, provided the original work is properly cited.

Copyright $\odot 2019$ The Journal of Minimally Invasive Surgery. All rights reserved.

\section{INTRODUCTION}

Appendectomy for acute appendicitis is one of the most commonly performed operations in the general surgery field, and the laparoscopic technique is considered the gold standard. ${ }^{1}$ The advantages of the laparoscopic technique include reduced postoperative pain and hospitalization duration, early return to routine activities, and a definite cosmetic benefit over the open approach. ${ }^{2}$

Single-incision laparoscopic appendectomy (SILA) has been proposed as an evolutionary step in minimal invasive surgery, and recent systemic reviews and pooled analyses have demonstrated that SILA is comparable to conventional laparoscopic appendectomy for acute appendicitis in adults in terms of operation time, length of postoperative stay, pain scores, and conversion or complication rates. ${ }^{3}$ A meta-analysis of ran- 
domized controlled trials comparing SILA and conventional laparoscopic appendectomy showed that SILA is comparable to conventional laparoscopic appendectomy in selected patients, although SILA takes a longer time, and is more technically demanding., ${ }^{4,5}$

The current practice of residents performing appendectomies is known to be safe and is not associated with a higher incidence of complications. ${ }^{6}$ The operative duration and complication rates were significantly reduced with the increased experience of residents. ${ }^{7.8}$ Recently, it has been observed that SILA by a surgical trainee could be performed safely with good postoperative outcomes and short learning curves. ${ }^{9} 10$ Moreover, SILA could be a useful teaching procedure for resident doctors to perform in selected patients, under the guidance of an experienced staff surgeon. ${ }^{11}$

The aim of this study was to report our experience with SILA performed by a resident, and to evaluate its safety and feasibility, along with a comparison of outcome with the procedures being performed by a staff surgeon.

\section{MATERIALS AND METHODS}

We conducted a retrospective case series analysis of 60 consecutive patients who underwent SILA at our institution between July 2016 and May 2018. The diagnosis of appendicitis was made based on physical examination and laboratory findings and was confirmed by ultrasound and abdominopelvic CT scan. The SILA technique was selected as per patient preference, and preoperative written informed consent was obtained. The inclusion criteria were as follows: a diagnosis of acute appendicitis including suppurative appendicitis, complicated appendicitis-like gangrenous appendicitis, and perforated appendicitis. Exclusion criteria were periappendiceal abscess, phlegmons requiring extensive operation such as ileocecectomy and right hemicolectomy, appendiceal tumor, and appendiceal carcinoma. Two surgeons, one an attending staff surgeon and the other a second-year resident, performed SILA. SILA performed by the resident was intraoperatively guided and supervised by a staff surgeon who is a board-certified colorectal surgeon, had completed fellowship training, and had performing routinely SILA in acute appendicitis and laparoscopic procedures for over 10 years. The selection of the operator was made according to confirmation by the attending surgeon of the preoperative radiologic examination.

Preoperative intravenous prophylactic antibiotics (1.0 g ceftriaxone) were administered from the diagnosis of acute appendicitis until postoperative day 1. After operation, feeding advanced from sips of water to regular diet, and was tolerated with encouragement of active ambulation. Postoperative pain was controlled by intramuscular injection of nonsteroidal anti- inflammatory drugs (NSAIDs) at the patient's request. Patients were discharged on postoperative day 2 after good postoperative recovery was ensured.

After approval from the Institutional Review Board, demographic data including age, sex, body mass index $\left(\mathrm{kg} / \mathrm{m}^{2}\right)$, American Society of Anesthesiologists (ASA) score, previous operation history, operation time, estimated blood loss, rate of open conversion, additional trocar insertion, complications (if any), pathology, time for oral intake, and duration of postoperative hospital stay were collected and compared between the resident- and staff-performed SILA groups.

\section{Surgical technique}

Under general anesthesia with the patient in a supine position, painting and draping was done. An umbilical incision of $1.5 \sim 2 \mathrm{~cm}$ was made, and the abdomen was entered by the open technique. A four-channeled single access system port (Nelis, Seoul, Korea) was inserted, and a pneumoperitoneum with 8 12 $\mathrm{mm} \mathrm{Hg} \mathrm{CO}$ pressure was created. A 30-degree 10$\mathrm{mm}$ optical scope, conventional straight laparoscopic instruments, and laparoscopic ultrasonic shears were used for SILA. The patient's position was changed to right side-up tilted, and single-incision laparoscopic exploration and manipulation was done to trace the ileocecal fold and inflamed appendix. The appendix or mesoappendix was grasped and pulled upward, the mesoappendix was dissected, and the appendiceal artery was ligated with ultrasonic shear coagulation. Intracorporeal double ligation of the appendix using loop-type 2-0 Polysorb pre-tied ligating loop (SurgiTie ${ }^{\mathrm{TM}}$, Covidien Ltd, USA) was performed, and the appendix was transected and removed with the ultrasonic shear. The specimen was delivered via the umbilical port and trapped in the vinyl pouch of the port. A closed suction drain was applied in the area to the right lower side of the operation field as per the decision of the operating or supervising surgeon. The abdominal wall was closed layer by layer in the usual manner with 2-0 vicryl.

\section{Statistical analysis}

Categorical data are shown as percentages; quantitative data are presented as median values with range. The student's t-test, chi-square test, and Fisher's exact test were used for statistical analysis. A $p$ value $<0.05$ was considered statistically significant. The SPSS software version 19.0 (SPSS for Windows, Advanced Statistics, Inc., Chicago, IL) was used.

\section{RESULTS}

In the present study, a total of 60 case-matched patients 
Table 1. Patient demographics

\begin{tabular}{lccc}
\hline & Resident SILA $(\mathbf{n = 3 0})$ & Staff SILA $(\mathbf{n}=30)$ & $p$ value \\
\hline Mean age (years), median (range) & $41.0(20 \sim 68)$ & $34.5(18 \sim 60)$ & 0.078 \\
Sex (M/F) & $12 / 18$ & $17 / 13$ & 0.196 \\
Body mass index, $\mathrm{kg} / \mathrm{m}^{2}$, median (range) & $22.6(17.3 \sim 20.1)$ & $23.4(19.5 \sim 28.9)$ & 0.624 \\
ASA score & & & \\
$1 / 2$ & $18 / 12$ & $20 / 10$ & 0.592 \\
Pathologic findings & & & 0.243 \\
Suppurative appendicitis & $24(80 \%)$ & $20(66.7 \%)$ & \\
Complicated appendicitis & $6(20 \%)$ & $10(33.3 \%)$ & \\
\hline
\end{tabular}

$\mathrm{BMI}=$ body mass index; $\mathrm{ASA}=$ American Society of Anesthesiologists, SILA = single-incision laparoscopic appendectomy .

with acute appendicitis underwent SILA by either a surgical resident or by an attending staff surgeon. There were no differences in age, sex ratio, BMI, ASA score, and pathologic findings of suppurative appendicitis and complicated appendicitis between the groups. Table 1 shows the demographics of patients in each group.

Mean operation time was longer in the resident group than the staff group ( $43.2 \pm 6.0$ minutes vs. $32.9 \pm 10.5$ minutes, $p<0.001)$. The operation time of the first 10 cases did not differ significantly from that of the third 10 cases in the resident group (46.3 \pm 5.6 minutes vs. $40.9 \pm 6.9$ minutes, $p=0.069$ ). The operation time of the resident group showed a decreasing tendency as the cases accumulated (Fig. 1).

An additional 5-mm trocar was placed in the right lower quadrant in three patients who underwent complex procedures. There were no significant differences in additional port insertion, drain insertion, and postoperative measured incision lengths between the two groups. No conversion to open procedure was found in either group. The operative data have been summarized in Table 2. Postoperative pain scores at 12 , 24, and 48 hours were similar in both groups. Tramadol 100 $\mathrm{mg}$ or ketorolac tromethamine $30 \mathrm{mg}$ was used for postoperative pain control, and the number of NSAID analgesic doses used after surgery was not different between the two groups.

No differences were found in time of onset of oral intake and postoperative hospital stay duration. The complication of surgical site infection was noted in two patients in the resident group, and one patient in the staff group, both of which involved the superficial incision area. They were managed with conservative treatment.

\section{DISCUSSION}

Herein, we report our early experience of SILA performed by a resident trainee. SILA performed by a resident under

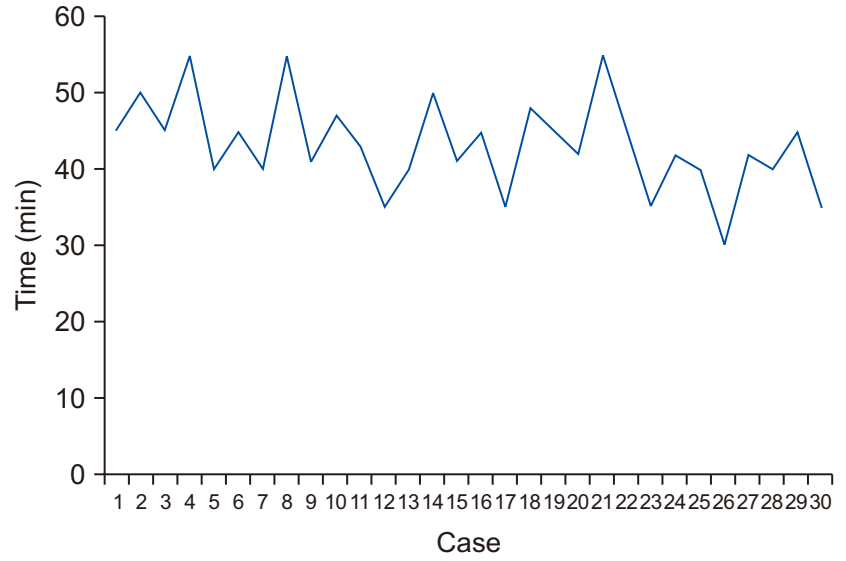

Fig. 1. The operation time of the resident group showed a decreasing trend as the cases accumulated.

supervision of an attending staff surgeon appears to be safe and feasible with comparable operation times, postoperative outcomes including length of hospital stay, and complication rates.

The operation time was longer in the resident group. However, intraoperative monitoring and supervision by an attending surgeon may not only prevent intraoperative events and perioperative complications but may also facilitate the perioperative surgical outcomes of the resident group, including operation time, and guarantee patients' safety. Data for the duration of hospital stay and rates of complication were comparable between the resident and attending groups. Jolley et al. showed that involvement of less experienced residents in laparoscopic procedures, such as appendectomy, might increase operation times and length of hospital stay, although it did not worsen clinical outcomes. ${ }^{8}$ They reported that resident performed operations do appear to have consequences in patients which may financially impact the healthcare system. In their 
Table 2. Operative results

\begin{tabular}{lccc}
\hline & Resident SILA (n=30) & Staff SILA (n=30) & $p$ value \\
\hline Operative time (min), median (range) & $45.0(30 \sim 55)$ & $30.0(20 \sim 60)$ & $<0.001$ \\
Additional port insertion & $1(3.3 \%)$ & $2(6.7 \%)$ & 0.554 \\
Conversion to open procedure & 0 & 0 & \\
Drain insertion & $3(10 \%)$ & $1(3.3 \%)$ & 0.301 \\
Postoperative incision length (cm), median (range) & $1.8(1.2 \sim 2.2)$ & $1.7(1.1 \sim 2.0)$ & 0.229 \\
Postoperative pain on resting (VAS 1 10) & & & \\
12 hours, median (range) & $5.2(4 \sim 6)$ & $5.0(4 \sim 6)$ & 0.451 \\
24 hours, median (range) & $3.8(2 \sim 5)$ & $3.7(2 \sim 6)$ & 0.665 \\
$\quad 48$ hours, median (range) & $2.1(2 \sim 5)$ & $2.0(2 \sim 5)$ & 0.562 \\
Postoperative numbers of analgesics used & & & 0.281 \\
1st day, median (range) & $1.4(1 \sim 2)$ & $1.3(1 \sim 2)$ & 0.527 \\
2nd day, median (range) & $0.2(0 \sim 1)$ & $0.2(0 \sim 1)$ & 0.694 \\
Time until oral feeding (days), median (range) & $1.1(1 \sim 2)$ & $1.1(1 \sim 2)$ & 0.064 \\
Length of postoperative hospital stay (days), median (range) & $2.9(2 \sim 4)$ & $2.6(2 \sim 4)$ & 0.554 \\
\hline Periumbilical surgical site infection & $2(6.7 \%)$ & $1(3.3 \%)$ & \\
\hline
\end{tabular}

SILA = single-incision laparoscopic appendectomy; VAS = visual analog scale.

report, resident involvement in laparoscopic appendectomy was associated with increased odds of readmission and longer operation times ( $>60$ minutes). Lin et al. evaluated outcomes and learning curves of laparoscopic appendectomy by residents. They concluded that operation time and complications could be reduced with increasing experience of the resident. Graat et al. concluded that appendectomy by residents is safe and not associated with a higher complication rate and that supervision by an experienced surgeon should be encouraged. ${ }^{6}$ Similar results have been previously reported showing that laparoscopic appendectomy can be safely performed by surgical residents without prior experience of open appendectomy, ${ }^{12}$ and that resident performed laparoscopic appendectomy was safe with no clinical impact with respect to complications. ${ }^{13}$ Hiramatsu et al. reported that laparoscopic appendectomy can be performed safely by surgical residents who had little experience or training with an animal model, or open appendectomy. ${ }^{12}$ In their literature, there were no statistical differences in demographics, conversion rate (0/174 vs. 1/90), operation time (75 minutes vs. 75 minutes), complications ( $7 \%$ vs. $4 \%$ ), and median length of hospital stay (4 days vs. 4 days). They insisted that in a laparoscopic era, laparoscopic appendectomy represents an important opportunity for training surgical residents, with little experience of open surgery. Lee et al. concluded that resident-performed laparoscopic appendectomy was safe, but was associated with significant prolongation in hospital stay and operation time, but not with complications. ${ }^{13}$

Regarding minimal invasive surgery, Vettoretto et al. reported in their systemic review that SILA could be an acceptable alternative to conventional laparoscopic appendectomy in the treatment of acute appendicitis, although the economic evaluation of the different techniques and apparatus for single access will only be evident after its wide spread clinical introduction. ${ }^{14} \mathrm{Xu}$ et al. stated that SILA took a longer time (43 mins vs. 38 mins, $p=0.0006$ ) and needed a greater number of extra trocars ( $7 \%$ vs. $0 \%, p<0.0001$ ), and that SILA is basically as feasible, effective, and safe as three port laparoscopic appendectomy. ${ }^{5}$ Clerveus et al. reported SILA to be comparable to conventional laparoscopic appendectomy in selected patients, with a higher technical failure rate (OR 3.30, 95\% CI 1.26 8.65, $p=0.01$ ) and longer operation time (mean difference 4.67, 95\% CI 1.76 7.57, $p=0.002){ }^{4}$. They evaluated cosmetic results, on short term follow up, interpretation of which should be done cautiously.

In the present study, SILA performed by the resident was supervised, and postoperative care was controlled by the attending surgeon. This might weaken the statistical power during analysis, although the variables in each group were not significantly different despite a longer operation time in the resident group. Wakasugi et al. reported a single center experience of SILA as a teaching procedure, wherein 77\% (156/202) of SILA were performed by resident doctors, indicating that it 
could be a useful teaching procedure for residents in selected patients, under the guidance of an experienced staff surgeon. ${ }^{10,11}$ In their report, the staff surgeons operated on more elderly patients with complicated appendicitis. Suh et al. have reported that surgical residents safely performed SILA with good postoperative outcomes. ${ }^{9}$ In the SILA group, the age was younger (26 years vs. 41 years, $p<0.005$ ), and the operation time (47.2 vs. $61.5 \mathrm{~min}, p<0.010$ ), and hospital stay (2.3 days vs. 2.7 days, $p=0.003$ ) were shorter than 3-port laparoscopic appendectomy. They reported a shorter learning curve of SILA by residents, measured by a mean operation time of below 48 minutes after about 10 cases of SILA.

The rate of additional port insertion was not significantly different between groups in the present study; although, it was noted that the staff surgeon operated on more complicated appendicitis cases.

Insertion of an additional port might be the bridge for overcoming the learning curve during SILA as a teaching procedure. This approach leads to a shorter operation time compared to the 3-port technique and improved cosmesis. ${ }^{15-17}$

In the present study, the surgical resident had assisted with various laparoscopic procedures such as cholecystectomy, three-port appendectomy, inguinal hernia, and gastrointestinal cancer operation. The intraoperative monitoring, supervision, and verbal guidance given by the attending surgeon may have shortened the learning curve and minimized perioperative events. Although the operation time increases, the operation performed by the resident under the supervision of the staff is necessary for resident education and training.

The present study has several limitations. It was a non-randomized, retrospective, case-cohort, single center study with a small sample size.

No significant differences in additional port insertion and pathological findings of appendicitis were found, although the patient selection after preoperative radiological imaging and intraoperative or perioperative guidance of the staff surgeon weakened the statistical power of our analysis owing to selection bias.

Further larger randomized studies are warranted.

\section{CONCLUSION}

Single incision laparoscopic appendectomies performed by residents are safe and feasible despite longer operation times. Perioperative supervision and guidance by an attending staff surgeon may facilitate surgical outcomes.

\section{CONFLICT OF INTEREST}

None.

\section{FUNDING}

None.

\section{ACKNOWLEDGMENTS}

None.

\section{REFERENCES}

1) Vettoretto N, Agresta F. A brief review of laparoscopic appendectomy: the issues and the evidence. Tech Coloproctol 2011;15:1-6.

2) Iqbal CW, Ostlie DJ. The minimally invasive approach to appendectomy: is less better? Eur J Pediatr Surg 2012;22:201-206.

3) Gill RS, Shi X, Al-Adra DP, Birch DW, Karmali S. Singleincision appendectomy is comparable to conventional laparoscopic appendectomy: a systematic review and pooled analysis. Surg Laparosc Endosc Percutan Tech 2012;22:319-327.

4) Clerveus M, Morandeira-Rivas A, Moreno-Sanz C, Herrero-Bogajo ML, Picazo-Yeste JS, Tadeo-Ruiz G. Systematic review and meta-analysis of randomized controlled trials comparing single incision versus conventional laparoscopic appendectomy. World J Surg 2014;38:1937-1946.

5) Xu AM, Huang L, Li TJ. Single-incision versus three-port laparoscopic appendectomy for acute appendicitis: systematic review and meta-analysis of randomized controlled trials. Surg Endosc 2015;29:822-843.

6) Graat LJ, Bosma E, Roukema JA, Heisterkamp J. Appendectomy by residents is safe and not associated with a higher incidence of complications: a retrospective cohort study. Ann Surg 2012;255: 715-719.

7) Lin YY, Shabbir A, So JB. Laparoscopic appendectomy by residents: evaluating outcomes and learning curve. Surg Endosc 2010; 24:125-130

8) Jolley J, Lomelin D, Simorov A, Tadaki C, Oleynikov D. Resident involvement in laparoscopic procedures does not worsen clinical outcomes but may increase operative times and length of hospital stay. Surg Endosc 2016;30:3783-3791.

9) Suh SG, Sohn HJ, Kim BG, et al. Single-Incision Laparoscopic Appendectomy by Surgical Trainees. Surg Laparosc Endosc Percutan Tech 2016;26:470-472.

10) Wakasugi M, Tsujimura N, Nakahara $Y$, et al. Single-incision laparoscopically assisted appendectomy performed by residents is safe and feasible: A single institution, retrospective case series. Ann Med Surg (Lond) 2017;15:43-46.

11) Wakasugi $M$, Tei $M$, Omori $T$, et al. Single-incision laparoscopic surgery as a teaching procedure: a single-center experience of more than 2100 procedures. Surg Today 2016;46:1318-1324.

12) Hiramatsu K, Toda S, Tate $T$, et al. Can laparoscopic appendectomy be safely performed by surgical residents without prior ex- 
perience of open appendectomy? Asian J Surg 2018;41:270-273.

13) Lee W, Park SJ, Park MS, Lee KY. Impact of Resident-Performed Laparoscopic Appendectomy on Patient Outcomes and Safety. J Laparoendosc Adv Surg Tech A 2018;28:41-46.

14) Vettoretto N, Cirocchi R, Randolph J, Morino M. Acute appendicitis can be treated with single-incision laparoscopy: a systematic review of randomized controlled trials. Colorectal Dis 2015;17: 281-289.

15) Golebiewski A, Losin M, Murawski M, Wiejek A, Lubacka D, Czauderna P. One, two or three port appendectomy - a rational approach. Wideochir Inne Tech Maloinwazyjne 2013;8:226-231.

16) Donmez T, Sunamak O, Ferahman S, Uludag SS, Yildirim D, Hut A. Two-port laparoscopic appendectomy with the help of a needle grasper: better cosmetic results and fewer trocars than conventional laparoscopic appendectomy. Wideochir Inne Tech Maloinwazyjne 2016;11:105-110.

17) Panait L, Bell RL, Duffy AJ, Roberts KE. Two-port laparoscopic appendectomy: minimizing the minimally invasive approach. J Surg Res 2009;153:167-171. 